\title{
First Principle Study of Salinity Measurement by 2D Material
}

\author{
Fan Jiang $\mathbb{D}^{1}{ }^{1}$ Bo Hu $\mathbb{D},{ }^{1}$ Weiguang Jia $\mathbb{D},{ }^{1}$ and Yi Zhou $\mathbb{D}^{2}$ \\ ${ }^{1}$ National Center of Ocean Standards and Metrology, Tianjin 300111, China \\ ${ }^{2}$ School of Mathematics and Physics, Qingdao University of Science and Technology, Qingdao 266061, China \\ Correspondence should be addressed to Weiguang Jia; 13752304783@163.com and Yi Zhou; zhouyi@qust.edu.cn
}

Received 7 May 2021; Revised 3 August 2021; Accepted 5 August 2021; Published 18 August 2021

Academic Editor: Ibrahim Alarifi

Copyright $\odot 2021$ Fan Jiang et al. This is an open access article distributed under the Creative Commons Attribution License, which permits unrestricted use, distribution, and reproduction in any medium, provided the original work is properly cited.

\begin{abstract}
By using first principle calculations, a simple model of salinity sensor based on graphene electrode is constructed and its electron transport property is systematically investigated. It is found that all saltwater clusters at different salinity exhibit an obvious increase of the current while the saltwater to be detected is passing through the device. Moreover, only changing one Na atom acted as the conductive medium, and the electron transport behaviors could be clearly distinguished among the saltwater by negative differential resistance phenomenon, which demonstrates that the graphene-based salinity sensor could be capable of distinguishing saltwater at different salinity efficiently and accurately. This study provides a new path for the creation of the novel salinity sensor by graphene and other $2 \mathrm{D}$ material electrode.
\end{abstract}

\section{Introduction}

It has been attracted more attention since the stable, oneatom layer graphene was fabricated in 2004 by exfoliating from its van der Waals solid graphite [1]. From then on, the atomic-scale thickness two-dimensional van der Waals layered materials started to intrigue a research boom because of their extremely large surface area [2-4] and through chemical vapor deposition (CVD) and other process methods, many graphene-like 2D nanomaterials have been fabricated with the similar hexagonal structure, such as boron nitride (h-BN) and transition-metal dichalcogenides (TMDCs) [3, $5,6]$. Due to quantum confinement effect, they exhibit marvelous electrical, optical, mechanical, and chemical properties. Based on these unique electronic properties, they have broad application prospects in electronics, optoelectronics, energy, environment, and other fields [2-6]. As a result of its wide bandgap insulating property, h-BN can be widely applied in nonlinear optics and ultraviolet lasers/detectors [7]. TMDCs have very rich electrical properties and a direct bandgap, making them show great potential for applications in areas such as optoeletronics and nanoelectronics [8].

Although various novel graphene-like two-dimensional materials have inspired a new wave of research and been studied experimentally and theoretically [9-12], graphene is the most popular candidate for the next generation of nanodevices because of its extremely high electron mobility which is beyond $200,000 \mathrm{~cm}^{2} \mathrm{~V}^{-1} \mathrm{~s}^{-1}$ at electron densities of about $2 \times 10^{11} \mathrm{~cm}^{-2}[13,14]$ while many other $2 \mathrm{D}$ materials do not possess. Furthermore, compared with other twodimensional materials, it possesses superior mechanical strength and flexibility [15]. Therefore, graphene is the most popular choice to develop the electrodes of the sensor owing to the large surface area and semimetallic property. Another advantage for sensing purpose is that graphene has less impact on the environment than other electrode materials [16]. Many of these properties lead the graphene to sensor applications, such as electrochemical and electrical sensors. In recent years, because of the higher electron mobility and surface activity, graphene has been largely considered as transistors for the detection of biomolecules and gas reducing response time and manufacture cost $[17,18]$. The high sensitivity of graphene to the local environment shows great advantage in sensing applications, where ultralow concentrations of adsorbed molecules react significantly to the electronic properties of graphene [19-22].

To date, detecting polluted gas and gaseous water molecules with graphene has been investigated extensively [2325]. Yokoyama et al. [23] found that at a higher voltage of $0.9 \mathrm{~V}$, suspended graphene sensor displayed the good 
selectivity to $\mathrm{H}_{2}$, and Leenaerts et al. [24] explored the graphene sensor adsorbed with various kinds of gas molecules theoretically which is consistent with the previous experimental work performed by Schedin et al. [25]. Salinity is the most important physical parameter in oceanography for understanding global climate and the dynamic feature of marine ecosystems, where differences in salinity drive ocean circulation [26]. Therefore, accurate salinity measurement is of great importance. Although the salinity sensor has been widely applied, there are still some problems to be solved. Slow reaction times of conductivity sensors which are installed in the glider produce erroneous salinities, especially on strong thermoclines, so the high sensitivity and miniaturization of the sensor are essential for accurate salinity measurements [27]. As 2D material exhibiting high conductivity and sensitivity, graphene is the most proper candidate to achieve accuracy and higher speed in detection where salinity sensing is required [20]. But detecting the salinity of the saltwater with the electrode of graphene has not been studied systematically yet. In this paper, we design a sensor with graphene electrode and perform a systematic study of the transport properties of a small drop of water with different salinity by using the Extended Huckel method and the nonequilibrium Green function.

\section{Materials and Methods}

Graphene nanoribbons perform different electronic properties; for the armchair edges, it shows semiconducting, and for the zigzag edges, it shows the metallic. Therefore, the graphene nanoribbons with the zigzag edges $(N=12)$ are chosen as the electrode of the salinity sensor, which is cut from the graphene. Many other researchers chose the field effect transistor- (FET-) based structure similar to ours as the sensor platform both in experiment and theory, and they found that using graphene as electrode could achieve accuracy and higher speed in detection $[20,21,28]$. All the dangling bonds at the edges are very unstable and tend to bond with other atoms in the vicinity, so carbon atoms at the edges are passivated by the hydrogen atoms to avoid the effects of dangling bonds. The structures are fully optimized to make sure that the fore on each atom is less than $0.5 \mathrm{eV} \AA^{-1}$.

Figure 1 shows the detected saltwater passing through the simulated devices in which the h-BN nanowalls are fabricated to allow the saltwater pass through the channel effectively. The water cluster is $20 \AA \times 10 \AA \times 5 \AA$ with 33 water molecules to ensure the simulated density of $1 \mathrm{~g} / \mathrm{cm}^{3}$. Recently, the researchers found that the minimal structure for proper solvation is made of 21 water molecules [29]. It is well known that almost $99 \%$ of the elements in seawater solutes are sodium $(\mathrm{Na})$ and chloride $(\mathrm{Cl})$. To simulate the saltwater simply, the $\mathrm{Na}$ atom is chosen for the solutes of simulated saltwater and the salinity of saltwater could be further deduced by calculating the $\mathrm{Na}$ concentration. The $\mathrm{Na}$ concentration is calculated by relative atomic mass in which $\mathrm{Na}$ atom is 23 and $\mathrm{H}_{2} \mathrm{O}$ is 18 . Therefore, the concentration of one, two, and three $\mathrm{Na}$ atoms in seawater solutes is $6 \%, 11 \%$, and $15 \%$, respectively. The water cluster is simulated enough time to an equilibrium state by dynamic simulation, and the trajectory recorded is $0.1 \mathrm{ps}$, which is repeated three times. The simulated water molecules in the middle of $z$ axis are substituted by $\mathrm{Na}$ atoms to ensure the solutes be detected effectively by graphene electrode in the channel. Figure 1(a) shows the top view of the device. The device consists of three parts: the left electrode, the central scattering region, and the right electrode, in which the width of electrodes is $0.43 \mathrm{~nm}$ $\times 3$ and central region is $0.43 \mathrm{~nm} \times 5$. The length of the device is $44.8 \mathrm{~nm}$, and the gap width of channel is $5.9 \mathrm{~nm}$ which allows the saltwater to pass freely through. Calculations of the electron transport properties were carried out in the bipolar system using the Extended Huckel method and the nonequilibrium Green function (NEGF) in the AtomistixToolKit (ATK) calculation package [30, 31], which only considers the electron-electron and not the electronphonon interaction. However, for graphene electrode, this method is enough to study the electron transport properties since it is consistent with the experimental work [32]. The set of Cerda parameters applies to carbon and hydrogen, where the vacuum is set to $-7.36577 \mathrm{eV}$ and $-6.2568 \mathrm{eV}$, respectively. Density mesh cut-off is $100 \mathrm{Ry}$, and electron temperature is $300 \mathrm{~K}$. The maximum interaction range is set to be $10 \AA$. The criterion of electron energy convergence is $10^{-5} \mathrm{eV}$. The current is calculated by integrating a transmission function using the Landauer formula.

\section{Results and Discussion}

Figure 2 shows the simulated current-voltage (I-V) characteristics of the pure water drop and saltwater with different $\mathrm{Na}$ concentration. It is noticed that when the bias is applied, there is no current in the transport direction for the pure water drop. However, all the saltwater clusters result in the onset of the current where the negative differential resistance (NDR) phenomenon is observed. As the pure water cluster is added with $\mathrm{Na}$ atom, the current initially increases with bias and then fluctuates slightly around the state of equilibrium between the current of 0.3 and $0.5 \mathrm{nA}$ before $0.4 \mathrm{~V}$. When the bias voltage is further increased and exceeds $0.4 \mathrm{~V}$, the current breaks the stable state and begins to come up. It is clear that in all three cases, the current climbs significantly as the bias voltage reaches $0.6 \mathrm{~V}$ and the peak currents are observed. Before that the growth trend becomes divided among the three cases, for the saltwater with $15 \% \mathrm{Na}$, the current ascends directly to the peak, while for the other two cases, the current increases fast first and slowly afterwards, even more approaching a peak value. The maximum obtained value of current for the saltwater with $6 \%, 11 \%$, and $15 \% \mathrm{Na}$ is $1.65 \mathrm{nA}, 1.96 \mathrm{nA}$, and $2.35 \mathrm{nA}$, respectively. Further increase in the bias voltage gives rise to a current drop until the bias of $0.7 \mathrm{~V}$. Similar to the growth tendency, except the case with $15 \% \mathrm{Na}$, the others drop quickly to a low value where the saltwater with $6 \% \mathrm{Na}$ (only one $\mathrm{Na}$ atom) results in the current maintaining the value of $0.3 \mathrm{nA}$. For the saltwater with $15 \% \mathrm{Na}$, the current declines less than half which still remains the high value among all the cases. In the rest time, the cases with $11 \%$ and $15 \% \mathrm{Na}$ appear a tight range and then the current continues to grow. The minimum current obtained for the saltwater with $6 \%$, 


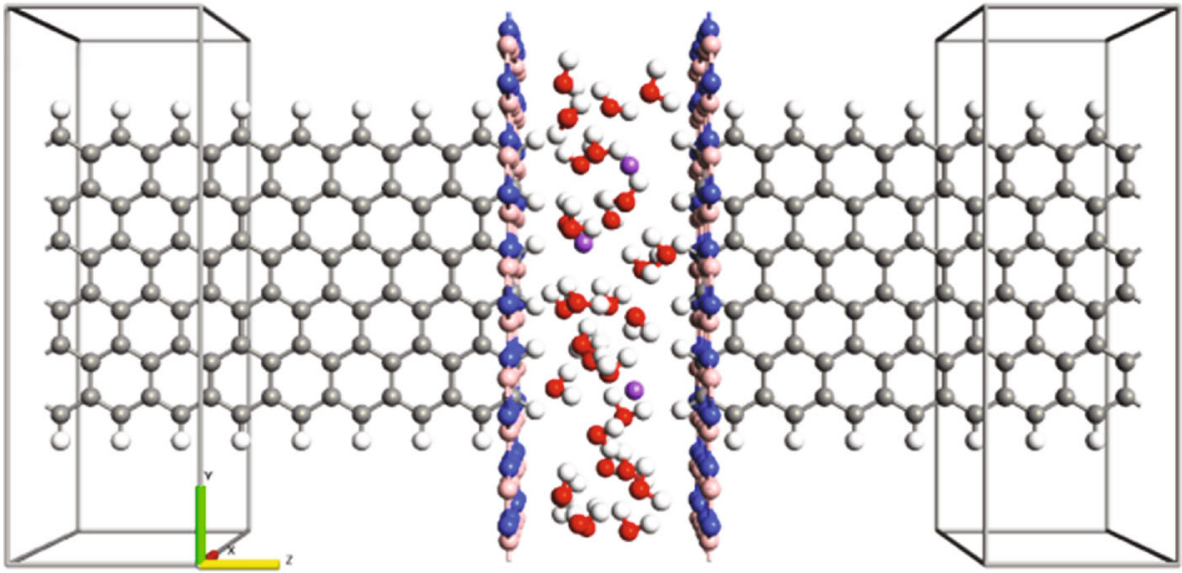

(a)

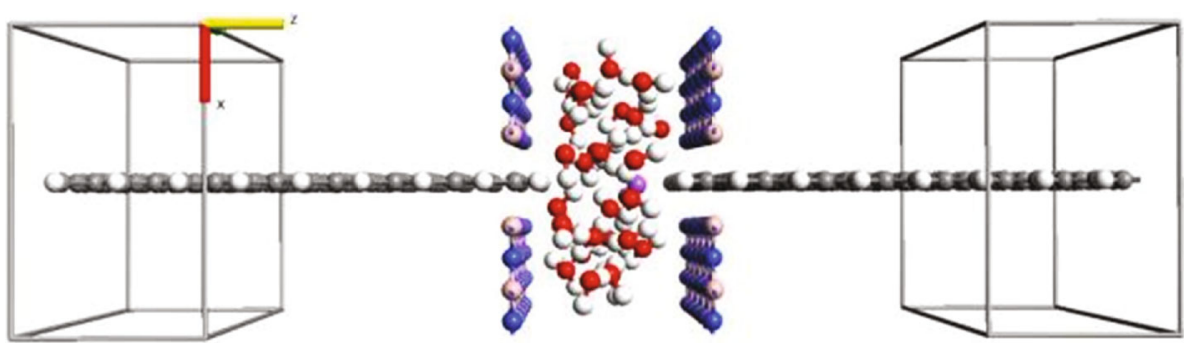

(b)

Figure 1: Detected saltwater as it passes through the simulated devices with three $\mathrm{Na}$ atoms at the channel: (a) top view and (b) side view. Hydrogen is white, oxygen is red, boron is pink, nitrogen is blue, carbon is gray, and sodium is purple.

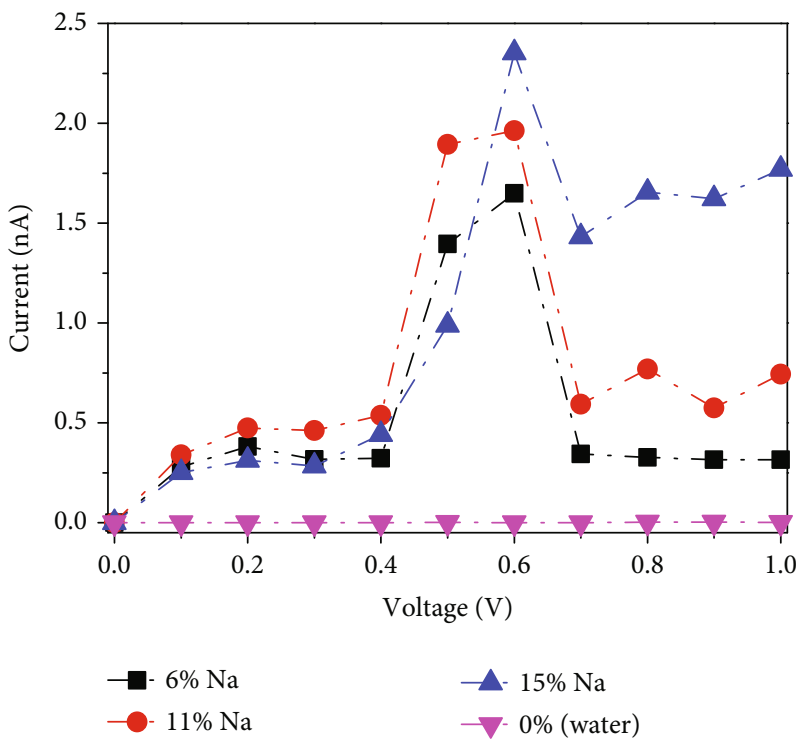

FIgURE 2: The current-voltage characteristics of the pure water drop and saltwater with different $\mathrm{Na}$ concentration.

$11 \%$, and $15 \% \mathrm{Na}$ is $0.34 \mathrm{nA}, 0.59 \mathrm{nA}$, and $1.43 \mathrm{nA}$ individually. Therefore, an obvious NDR behavior appears in all the cases, and the peak-to-valley ratio (PVR) is calculated to compare the difference. The obtained PVR is 4.9, 3.3, and 1.6 for the saltwater with $6 \%, 11 \%$, and $15 \% \mathrm{Na}$, respectively. According to the above analysis, it is clear that the saltwater with different $\mathrm{Na}$ concentration shows obvious differences in I-V characteristics; especially from the pure water to seawater, the current undergoes a dramatic change, which demonstrates that the graphene electrode is extremely sensitive to the change of the salinity. As mentioned above, through the simple saltwater models, the salinity could be deduced and the salinity of the detected saltwater could match the I-V characteristics, that is, the measurement principle of conductivity sensor in the salinometer. The peak, valley current, and PVR are all could be the parameters of the conductivity sensor because there are significant differences in these obtained values for the saltwater with different $\mathrm{Na}$ concentration. Moreover, the conductivity parameters differ obviously when altering one solute atom, indicating that the sensor with graphene electrode is a salinity sensor with high accuracy. In the follow-up works, we will consider the electron-phonon interaction and study the thermal conduction of the $2 \mathrm{D}$ materials by machine-learning interatomic potentials [33, 34].

In order to gain further insight into the parameters which shows the difference in the I-V characteristics among the cases with $\mathrm{Na}$ atom, transmission spectrum at some bias voltages is plotted in Figure 3. The transmission spectrum is influenced by both energy and bias voltage which indicates the probability of an electron transporting through the channel in certain energy. When a bias voltage is applied, the current can be calculated by the Landauer formula [35]:

$$
I\left(V_{b}\right)=\frac{2 e}{h} \int T\left(E, V_{b}\right)\left[f_{R}\left(E, V_{b}\right)-f_{L}\left(E, V_{b}\right)\right] d E,
$$




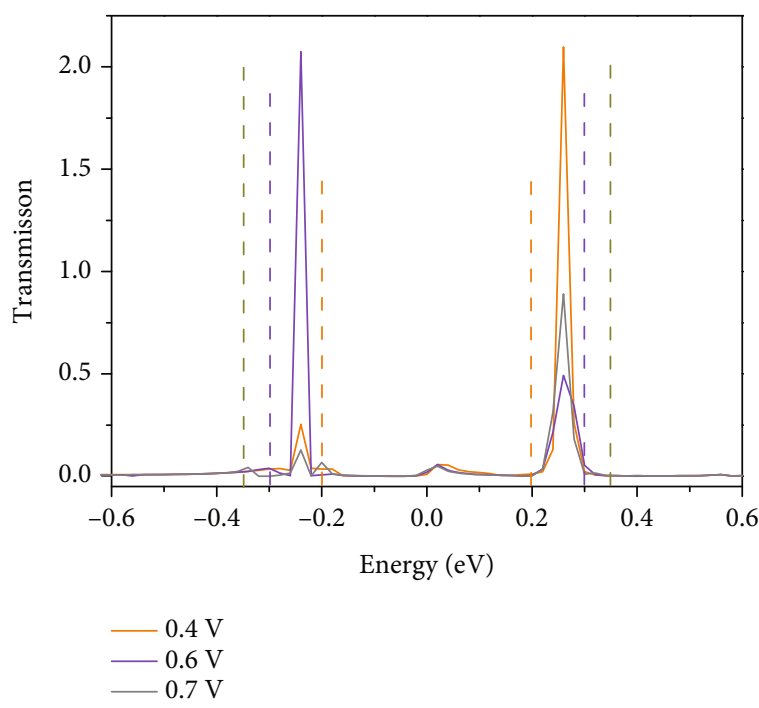

(a)

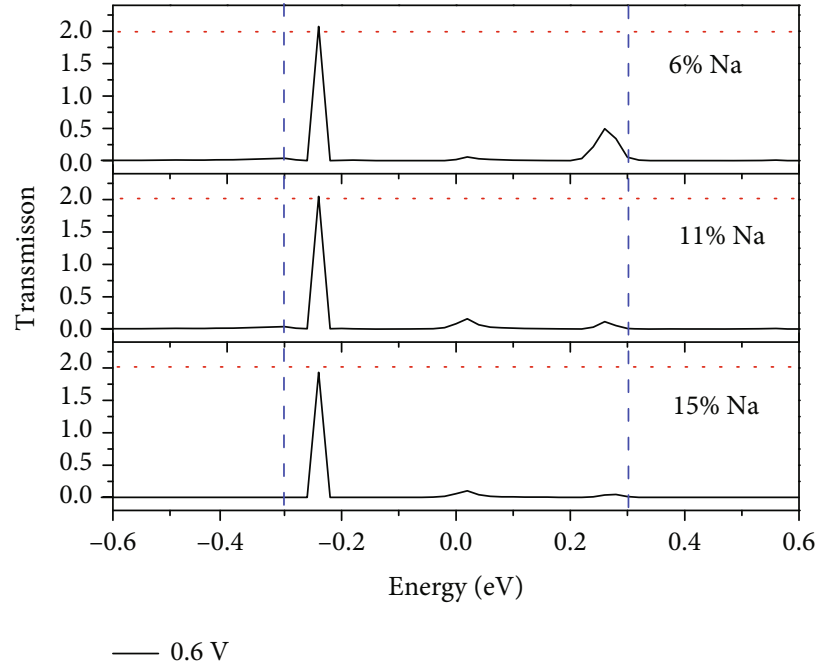

(b)

Figure 3: Transmission spectrum of the saltwater (a) with $15 \% \mathrm{Na}$ at $0.4 \mathrm{~V}, 0.6 \mathrm{~V}$, and $0.7 \mathrm{~V}$ and (b) with different Na concentration at $0.6 \mathrm{~V}$.

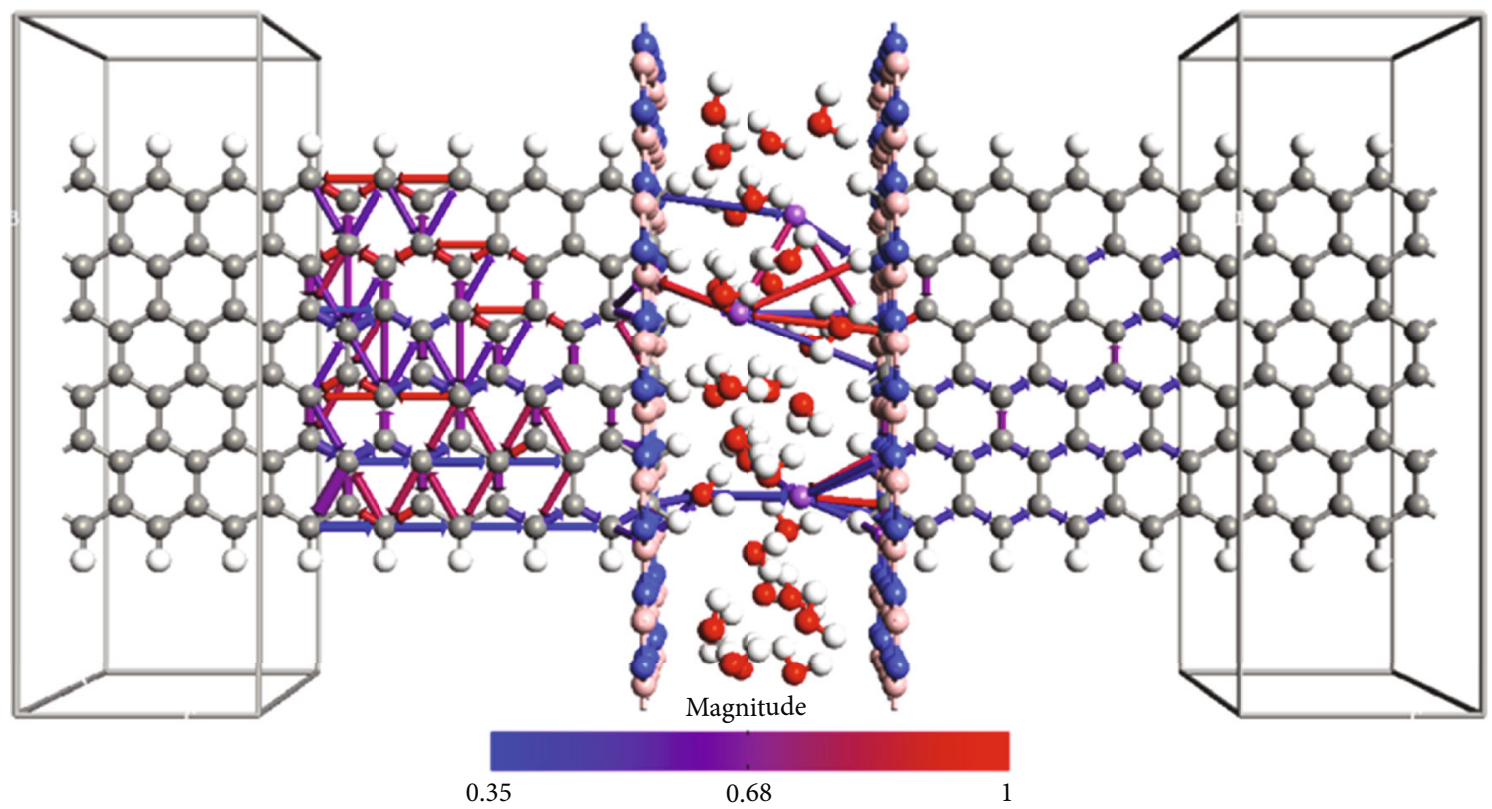

Figure 4: Transmission pathways of saltwater with $15 \% \mathrm{Na}$ at $0.6 \mathrm{~V}$ at energy $-0.24 \mathrm{eV}$. Hydrogen is white, oxygen is red, boron is pink, nitrogen is blue, carbon is gray, and sodium is purple.

where $V_{b}$ is the bias voltage, $T\left(E, V_{b}\right)$ is the transmission probability, and $f_{R}\left(E, V_{b}\right)$ and $f_{L}\left(E, V_{b}\right)$ are Fermi-Dirac distribution function of the right and left electrodes.

According to the Landauer formula, the current is calculated by integrating the transmission probability in the bias window, indicating that the transmission spectrum going to the bias window mainly contributes to the current. To understand the NDR effect, the case with $15 \% \mathrm{Na}$ is taken for an example, as shown in Figure 3(a). At the bias of $0.4 \mathrm{~V}$, the transmission spectrum exhibits two obvious peaks at $0.26 \mathrm{eV}$ and $-0.24 \mathrm{eV}$. As the peaks are not in the bias window, they could not contribute to the current resulting in a low current. However, when the bias is increased to $0.6 \mathrm{eV}$, there is also a dominant peak at $-0.24 \mathrm{eV}$ and another smaller peak at $0.26 \mathrm{eV}$, but the two peaks locate inside the bias window leading to a peak current. At $0.7 \mathrm{~V}$, the transmission probability still shows two peaks with the same energy where the peak value at $-0.24 \mathrm{eV}$ drops significantly and almost increases double at $0.26 \mathrm{eV}$ compared to the bias of $0.6 \mathrm{eV}$, resulting a valley current which is higher than that of the bias of $0.4 \mathrm{eV}$. Next, peak current is taken for exhibiting the difference in I-V characteristics among the models with different $\mathrm{Na}$ concentration by transmission spectrum. As can be seen from Figure 3(b), they all exhibit a dominant peak at energy 


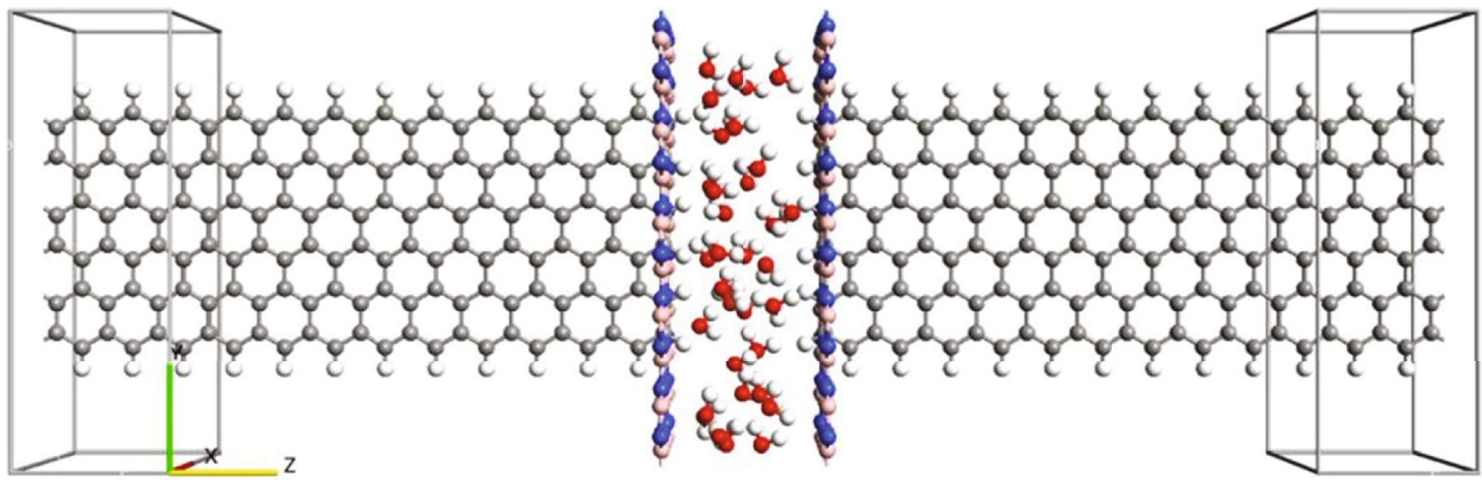

(a)

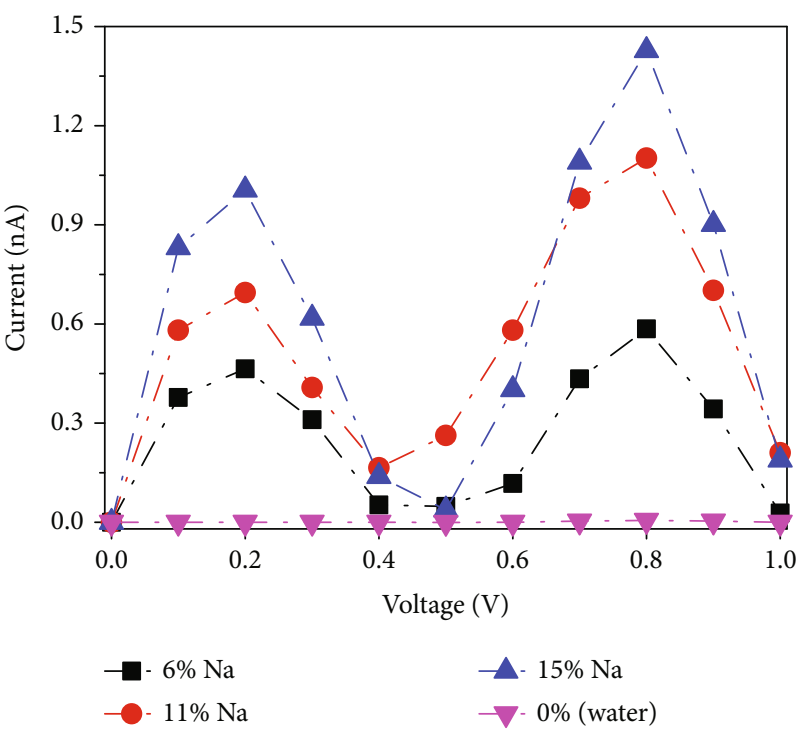

(b)

Figure 5: (a) Top view of detected pure water as it passes through the sensor with longer graphene electrode and (b) the current-voltage characteristics of the saltwater with different $\mathrm{Na}$ concentration. Hydrogen is white, oxygen is red, boron is pink, nitrogen is blue, and carbon is gray.

$-0.24 \mathrm{eV}$ and small peaks at other energies inside the bias window. Although the dominant peak grows a little with the increase of $\mathrm{Na}$ concentration, it contributes significantly to the peak current, especially the saltwater with the concentration of $\mathrm{Na}$ from $6 \%$ to $11 \%$. At the energy $0.26 \mathrm{eV}$, the contribution of small peak to current is increased with the higher growth in transmission spectrum. It can be seen from the above analysis that the current peak is driven by the dominant transmission peak at $-0.24 \mathrm{eV}$.

Figure 4 shows the transmission pathways of saltwater with $15 \% \mathrm{Na}$ at $0.6 \mathrm{~V}$ at energy $-0.24 \mathrm{eV}$. The transmission pathways describe the paths of electrons transporting through the device where the arrow represents the direction and the color indicates the relative magnitude of the transmission probability. The transmission pathways are stronger at the left hand of the device than those at the right side. The pathways also occur in the saltwater cluster between the graphene electrodes where the $\mathrm{Na}$ atoms provide the transport paths in the saltwater cluster. And this is consistent with the above analysis that only the pure water cluster could not generate current, and the current is enhanced obviously by adding $\mathrm{Na}$ atom.

In the end, we construct a sensor device with longer graphene electrode in which the size of channel and Na concentration is the same with the abovementioned device, as shown in Figure 5(a). I-V characteristics of the pure water and saltwater with different $\mathrm{Na}$ concentration are calculated (Figure 5(b)). It is also found that pure water could not generate the current, and there is NDR effect in all the saltwater clusters. Among all the cases of different $\mathrm{Na}$ concentration, it also results in significant differences in I-V characteristics. Therefore, no matter the graphene electrode is long or short, the graphene sensor is sensitive to the $\mathrm{Na}$ concentration.

\section{Conclusions}

Using the nonequilibrium Green function together with the Extended Huckel method, a simple model of salinity sensor based on graphene electrode is constructed and the electron transport property of the simulated saltwater is investigated. 
It is found that except the pure water, all the saltwater clusters exhibit an obvious increase of the current and the NDR effect while the detected saltwater is passing through the device, because the $\mathrm{Na}$ atom acts as a conductive medium in the simulated saltwater cluster. Moreover, only changing one $\mathrm{Na}$ atom acted as the conductive medium, and the I-V characteristics could be clearly distinguished among the saltwater by peak current, valley current, and the obtained PVR, no matter the graphene electrode is long or short. This study may provide a new path for the creation of the novel salinity sensor by graphene and other $2 \mathrm{D}$ materials electrode.

\section{Data Availability}

The data used to support the findings of this study are included within the article.

\section{Conflicts of Interest}

The authors declare that there is no conflict of interests regarding the publication of this paper.

\section{Acknowledgments}

The authors would like to acknowledge the support from the National Natural Science Foundation of China (11804179) and Natural Science Foundation of Shandong Province (ZR2019PA018).

\section{References}

[1] K. S. Novoselov, A. K. Geim, S. V. Morozov et al., "Electric field effect in atomically thin carbon films," Science, vol. 306, no. 5696, pp. 666-669, 2004.

[2] M. Long, P. Wang, H. Fang, and W. Hu, "Progress, challenges, and opportunities for 2D material based photodetectors," Advanced Functional Materials, vol. 29, no. 19, article 1803807, 2019.

[3] X. Cai, Y. Luo, B. Liu, and H.-M. Cheng, "Preparation of 2D material dispersions and their applications," Chemical Society Reviews, vol. 47, no. 16, pp. 6224-6266, 2018.

[4] T. Tan, X. Jiang, C. Wang, B. Yao, and H. Zhang, "2D material optoelectronics for information functional device applications: status and challenges," Advanced Science, vol. 7, no. 11, article 2000058, 2020.

[5] A. Laturia, M. L. Van de Put, and W. G. Vandenberghe, "Dielectric properties of hexagonal boron nitride and transition metal dichalcogenides: from monolayer to bulk," $n p j 2 D$ Materials and Applications, vol. 2, no. 1, 2018.

[6] H. Zhang, M. Chhowalla, and Z. Liu, "2D nanomaterials: graphene and transition metal dichalcogenides," Chemical Society Reviews, vol. 47, no. 9, pp. 3015-3017, 2018.

[7] J. Zhang, B. Tan, X. Zhang et al., "Atomically thin hexagonal boron nitride and its heterostructures," Advanced Materials, vol. 33, no. 6, article 2000769, 2021.

[8] S. Manzeli, D. Ovchinnikov, D. Pasquier, O. V. Yazyev, and A. Kis, "2D transition metal dichalcogenides," Nature Reviews Materials, vol. 2, no. 8, 2017.

[9] S. Mao, J. Chang, H. Pu et al., "Two-dimensional nanomaterial-based field-effect transistors for chemical and biological sensing," Chemical Society Reviews, vol. 46, no. 22, pp. 6872-6904, 2017.

[10] A. N. Abbas, B. Liu, L. Chen et al., "Black phosphorus gas sensors," ACS Nano, vol. 9, no. 5, pp. 5618-5624, 2015.

[11] D. Sarkar, X. Xie, J. Kang et al., "Functionalization of transition metal dichalcogenides with metallic nanoparticles: implications for doping and gas-sensing," Nano Letters, vol. 15, no. 5, pp. 2852-2862, 2015.

[12] T. Xie, G. Xie, Y. Su, D. Hongfei, Z. Ye, and Y. Jiang, "Ammonia gas sensors based on poly (3-hexylthiophene)-molybdenum disulfide film transistors," Nanotechnology, vol. 27, no. 6, article 065502, 2016.

[13] K. I. Bolotin, K. J. Sikes, Z. Jiang et al., "Ultrahigh electron mobility in suspended graphene," Solid State Communications, vol. 146, no. 9-10, pp. 351-355, 2008.

[14] A. K. Geim and K. S. Novoselov, "The rise of graphene," Nature Materials, vol. 6, no. 3, pp. 183-191, 2007.

[15] E. Singh, M. Meyyappan, and H. S. Nalwa, "Flexible graphenebased wearable gas and chemical sensors," ACS Applied Materials \& Interfaces, vol. 9, no. 40, pp. 34544-34586, 2017.

[16] "Graphene sensors: introduction and market status," 2019, https://www.graphene-info.com/graphene-sensors.

[17] J. Wu, K. Tao, J. Miao, and L. K. Norford, "Three-dimensional hierarchical and superhydrophobic graphene gas sensor with good immunity to humidity," in 2018 IEEE Micro Electro Mechanical Systems (MEMS), pp. 901-904, Belfast, UK, 2018.

[18] A. Merkoçi, "Graphene-based biosensors," 2D Materials, vol. 7, no. 4, article 040401, 2020.

[19] D. J. Buckley, N. C. G. Black, E. G. Castanon, C. Melios, M. Hardman, and O. Kazakova, "Frontiers of graphene and 2D material-based gas sensors for environmental monitoring," 2D Materials, vol. 7, no. 3, article 032002, 2020.

[20] E. Akbari, R. Yusof, M. T. Ahmadi et al., "Bilayer graphene application on $\mathrm{NO}_{2}$ sensor modelling," Journal of Nanomaterials, vol. 2014, Article ID 534105, 7 pages, 2014.

[21] X. Li, J. Shi, J. Pang, W. Liu, H. Liu, and X. Wang, "Graphene channel liquid container field effect transistor as $\mathrm{pH}$ sensor," Journal of Nanomaterials, vol. 2014, Article ID 547139, 6 pages, 2014.

[22] Y. Jiang, S. Yang, S. Li, W. Liu, and Y. Zhao, "Highly sensitive CO gas sensor from defective graphene: role of van der Waals interactions," Journal of Nanomaterials, vol. 2015, Article ID 504103, 7 pages, 2015.

[23] T. Yokoyama, T. Tanaka, Y. Shimokawa, R. Yamachi, Y. Saito, and K. Uchida, "Pd-functionalized, suspended graphene nanosheet for fast, low-energy multimolecular sensors," ACS Applied Nano Materials, vol. 1, no. 8, pp. 3886-3894, 2018.

[24] O. Leenaerts, B. Partoens, and F. M. Peeters, "Adsorption ofH2O,NH3, CO,NO2, and $\mathrm{NO}$ on graphene: a firstprinciples study," Physical Review B, vol. 77, no. 12, 2008.

[25] F. Schedin, A. K. Geim, S. V. Morozov et al., "Detection of individual gas molecules adsorbed on graphene," Nature Materials, vol. 6, no. 9, pp. 652-655, 2007.

[26] R. Venkatesan, K. Ramesh, M. Arul Muthiah, K. Thirumurugan, and M. A. Atmanand, "Analysis of drift characteristic in conductivity and temperature sensors used in Moored buoy system," Ocean Engineering, vol. 171, pp. 151-156, 2019.

[27] Z. Liu, J. Xu, and J. Yu, "Real-time quality control of data from Sea-Wing underwater glider installed with Glider Payload 
CTD sensor," Acta Oceanologica Sinica, vol. 39, no. 3, pp. 130$140,2020$.

[28] P. Zhang, Y. Xiao, J. Zhang, B. Liu, X. Ma, and Y. Wang, "Highly sensitive gas sensing platforms based on field effect transistor-a review," Analytica Chimica Acta, vol. 1172, article 338575, 2021.

[29] A. Rognoni, R. Conte, and M. Ceotto, "How many water molecules are needed to solvate one?," Chemical Science, vol. 12, no. 6, pp. 2060-2064, 2021.

[30] K. Stokbro, D. E. Petersen, S. Smidstrup, A. Blom, M. Ipsen, and K. Kaasbjerg, "Semiempirical model for nanoscale device simulations," Physical Review B, vol. 82, no. 7, 2010.

[31] M. Brandbyge, J.-L. Mozos, P. Ordejón, J. Taylor, and K. Stokbro, "Density-functional method for nonequilibrium electron transport," Physical Review B, vol. 65, no. 16, 2002.

[32] P. D. Nguyen, T. C. Nguyen, F. M. Hossain, D. H. Huynh, R. Evans, and E. Skafidas, "Negative differential resistance effect in planar graphene nanoribbon break junctions," Nanoscale, vol. 7, no. 1, pp. 289-293, 2015.

[33] B. Mortazavi, E. V. Podryabinkin, S. Roche, T. Rabczuk, X. Zhuang, and A. V. Shapeev, "Machine-learning interatomic potentials enable first-principles multiscale modeling of lattice thermal conductivity in graphene/borophene heterostructures," Materials Horizons, vol. 7, no. 9, pp. 2359-2367, 2020.

[34] B. Mortazavi, I. S. Novikov, E. V. Podryabinkin et al., "Exploring phononic properties of two-dimensional materials using machine learning interatomic potentials," Applied Materials Today, vol. 20, article 100685, 2020.

[35] M. Büttiker, Y. Imry, R. Landauer, and S. Pinhas, "Generalized many-channel conductance formula with application to small rings," Physical Review B, vol. 31, no. 10, pp. 6207-6215, 1985. 